\title{
Alteraciones de la nutrición en la enfermedad renal
}

\author{
Dolores Andreu Périz, María del Carmen Moreno Arroyo, Miguel Ángel Hidalgo Blanco
}

Facultad de Medicina y Ciencias de la Salud. Universitat de Barcelona. España

Uno de los problemas que se plantea con mayor frecuencia a las personas sometidas a terapia sustitutiva de la función renal es nutrirse adecuadamente, siendo muy relevante la malnutrición, que es un importante predictor de morbimortalidad independientemente de la enfermedad de base ${ }^{1-2}$. En distintos estudios, se refleja que entre el 30 y el $70 \%$ de los pacientes en diálisis están malnutridos y se ha correlacionado la malnutrición con la mortalidad en cualquier forma de tratamiento sustititivo ${ }^{3-4}$. La malnutrición no solo puede observarse en una valoración general subjetiva, sino que puede objetivarse mediante distintos parámetros como los niveles de albúmina, prealbúmina, colesterol, BUN, creatinina y masa magra ${ }^{1-4}$.

Aunque las causas de malnutrición son muy numerosas, el principal factor es la disminución de la ingesta, jugando un papel importante la toxicidad uremica ${ }^{3-5}$. A estos pacientes se les suele prescribir dieta sin sal y pobre en potasio, con restricción de los líquidos, lo que la hace poco atractiva e interfiere en sus hábitos de vida, esto puede agravarse si coexiste con depresión 0 con condiciones socioeconómicas negativas ${ }^{1-6}$. Por otra parte, la insuficiencia renal conlleva alteraciones digestivas y hormonales que también favorecen la anorexia y tanto la hemodiálisis como la diálisis peritoneal provocan pérdida de nutrientes. También cabe destacar que la inflamación sistémica se asocia con frecuencia a malnutrición y arteriosclerosis, lo que se ha denominado síndrome MIA ${ }^{1}$.

\section{Correspondencia:}

Dolores Andreu Periz

Universidad de Barcelona.Departamento de Enfermería Fundamental y Medicoquirúrgica

C/ Feixa Llarga, s/n

08907 L'Hospitalet de Llobregat. Barcelona. España

E-mail:Iolaandreu@ub.edu
Para evitar la desnutrición es fundamental corregir y evaluar periódicamente el estado nutricional y corregir el desequilibrio metabólico mediante la diálisis adecuada y dar soporte nutricional con un consejo dietético individualizado, administrando suplementos orales cuando la ingesta de proteínas es inadecua$\mathrm{da}^{6,7}$. La importancia de seguir una dieta adecuada, que puede ser importante suplementar, el riesgo de limitar el consumo de proteínas o como se relaciona la nutrición en diferentes circunstancias del tratamiento, son importantes para la educación sanitaria del paciente renal, por lo que alguno de estos aspectos se recogen en los artículos siguientes.

Noce A, Vidiri MF, Marrone G, Moriconi E, Bocedi A, Capria A, et al. Is low-protein diet a possible risk factor of malnutrition in chronic kidney disease patients? Cell Death Discov. 2016 May 9; 2: 16026.

Enlentecer la progresión de la enfermedad renal es uno de los objetivos básicos en la atención del paciente y una de las herramientas más utilizadas en la terapia conservadora es la dieta baja en proteínas, aunque se ha de asumir el riesgo de un desgaste proteico-energético. El objetivo de este estudio es evaluar la posible correlación entre la dieta baja en proteínas recomendada al paciente renal y la desnutrición. En este estudio se incluyó a 41 pacientes con enfermedad renal crónica en fases III B / IV según las directrices de K-DIG0, que siguieron durante 6 semanas una dieta con la ingesta de proteína controlada, siendo la cantidad diaria recomendada de $0,7 \mathrm{~g} /$ por $\mathrm{kg}$ de peso corporal ideal/ día de proteína. Los pacientes mostraron una disminución significativa de los valores de albúmina de suero después de 6 semanas de dieta en comparación con los valores basales $(P=$ $0,039)$, mientras que la proteína C-reactiva aumentó significativamente respecto a la que mostraban antes de iniciar la dieta $(P=0,131)$. A partir del análisis de la composición corporal, se demostró una degradación significativa del porcentaje de masa libre de grasa al final del estudio $(p=0,0489)$, los autores relacionan 
este dato con un aumento total de agua corporal. El índice de masa celular, masa muscular y la masa celular corporal se redujeron significativamente después de las 6 semanas de dieta. Por último, tambien se encontró una reducción significativa en el ángulo de fase de la bioempedancia al final del estudio en comparación con los valores basales. Los autores concluyen que la dieta baja en proteínas ralentiza la progresión de la enfermedad renal, pero empeora el estado nutricional de los pacientes.

\section{Ipema KJ, Struijk $\mathbf{S}$, van der Velden $A$, Westerhuis $\mathbf{R}$, van der Schans CP, Gaillard CA, Krijnen WP, Franssen CF. Nutritional Status in Nocturnal Hemodialysis Patients - A Systematic Review with Meta-Analysis. PLoS One. 2016 Jun 20; 11(6):e0157621}

Esta revisión sistemática pretende responder la cuestión de si una dieta más liberal al incluir a los pacientes en hemodiálisis nocturna frecuente y por tanto eliminar más eficazmente los productos de desecho y líquidos, conduce a una mejor ingesta con la consiguiente mejoría del estado nutricional. Se llevó a cabo una búsqueda bibliográfica sistemática de las bases de datos MEDLINE, CINAHL, EMBASE y The Cochrane Library, para identificar estudios que trataran sobre el estado nutricional posterior al cambio de hemodiálisis convencional a nocturna y su efecto en la ingesta de proteínas, los índices de laboratorio del estado nutricional y la composición corporal. Se recogieron los datos de la albúmina, la tasa de catabolismo proteico, el peso corporal seco, el índice de masa corporal, el ángulo de fase, la ingesta de proteínas, y la ingesta calórica.

Este trabajo analiza 13 estudios con 282 pacientes que cambiaron de hemodiálisis convencional a nocturna, mientras que en la parte de la revisión correspondiente al meta-análisis se incluyeron 9 estudios en 229 pacientes. Los resultados que se obtuvieron en los 4 estudios con grupo control demostraron que la albúmina aumentó significativamente desde el inicio del cambio de hemodiálisis convencional a nocturna en 4-6 meses en comparación con los pacientes que permanecieron con hemodiálisis convencional (diferencia media de $1,3 \mathrm{~g} / \mathrm{l}$; IC del 95\%: 0,02; 2,58; $p=0,05)$. En estudios controlados de base, desde el inicio hasta 4-6 meses de tratamiento con hemodiálisis nocturna frecuente, se identificaron aumentos significativos en la albúmina, en la Tasa de Catabolismo Proteico y en la ingesta de proteínas y de calorías, mientras que los parámetros nutricionales no cambiaron significativamente. Se obtuvieron resultados simi- lares para la comparación entre el inicio y 8-12 meses de tratamiento con hemodiálisis nocturna frecuente.

Los autores afirman que la mayoría de los estudios revisados tuvieron tamaños de muestra moderados; algunos tenían registros incompletos de la dieta, era relativamente breve el período de seguimiento y diferían notablemente en lo que respecta su diseño.

Pese a estas reservas metodológicas los estudios analizados llevan a afirmar que la hemodiálisis nocturna frecuente aunque se asocia significativamente a una mayor ingesta de proteínas y de energía, así como a aumentos en la albúmina del suero y de la tasa de catabolismo proteico, no aporta datos concluyentes sobre cambios en la composición corporal.

\section{Martin-Alemany G, Valdez-Ortiz R, Olvera-Soto G Gómez-Guerrero I, Aguire-Esquivel G, Cantu- Quintanilla $G$ et al. Efects of resistance exercise and oral nutritional supplementation during hemodialysis on indicators of nutritional status and quality of life. Nephrol Dial Transplant. 2016 Aug 10. Pii: gfw297.}

Numerosos trabajos mencionan la importancia de los Suplementos Nutricionales Orales para evitar la malnutrición. Este estudio pantea como objetivo evaluar el efecto de los Suplementos Nutricionales Orales reforzado por Ejercicios de Resistencia, sobre los marcadores del estado nutricional y sobre la Calidad de Vida de los pacientes sometidos a hemodiálisis. En un ensayo clínico aleatorizado, 36 pacientes fueron divididos en dos grupos: un grupo control en el que los pacientes durante 12 semanas recibieron suplementos nutricionales en las sesiones de hemodiálisis y un grupo de intervención que recibió suplementos orales y además realizo sesiones de 40 min de ejercicios de resistencia en el transcurso de la hemodiálisis. Se registraron las medidas antropométricas, bioquímicas, dietéticas y bioeléctricas de impedancia de los pacientes, así como su calidad de vida evaluadas utilizando el Kidney Disease Quality of Life Short Form.

Al inicio, 20 pacientes presentaban pérdidas proteicas y energéticas según criterios de la Sociedad Internacional de Nutrición Renal y Metabolismo. Se encontraron cambios estadísticamente significativos en ambos grupos, tales como aumentos en el peso corporal, en el índice de masa corporal, en la circunferencia del brazo, en el pliegue cutáneo, en el porcentaje de masa grasa, en la fuerza de empuñadura, en el ángulo de fase y en la albúmina sérica. Se observó una 
disminución en la prevalencia de pérdidas proteicas y energéticas en ambos grupos al final de la intervención. Una comparación entre grupos no mostró ninguna diferencia estadísticamente significativa en los parámetros antropométricos y bioquímicos ni mejora significativa en la calidad de vida y composición corporal. La ingesta de energía y proteína aumentaron significativamente durante el período de estudio para todos los pacientes. A la vista de estos resultados, los autores consideran que la suplementación nutricional oral durante la hemodiálisis mejora el estado nutricional y el añadir ejercicios de resistencia durante la hemodiálisis no parece aumentar los efectos anabólicos agudos que acompañan a la desnutrición.

\section{Cheng TY, Tarng DC, Liao YM, Lin PC. Effects of systematic nursing instruction on a low- phosphorus diet, serum phosphorus level and pruritus of patients on haemodialysis. Clin Nurs. 2016 Jul 6. doi: 10.1111/jocn.13471.}

Uno de los problemas que se plantea en la enfermedad renal crónica es la hiperfosforemia y su relación con el prurito que a menudo limita la calidad de vida de los pacientes, por esto es necesario que los pacientes aprendan a controlar su ingesta de fosforo. Este reciente artículo demuestra una vez más la importancia de la función educadora de la enfermería, que en este trabajo plantea como objetivo demostrar la eficacia de la instrucción sistemática por parte de los profesionales respecto a una dieta baja en fósforo y esto es independiente de la cultura del país y de sus costumbres respecto a la alimentación. Un grupo de enfermeras de un centro médico en Taipéi, Taiwán, realizaron este trabajo experimental en el que 94 pacientes en programa de hemodiálisis se separaron en dos grupos. El grupo control recibió la instrucción tradicional de enfermería respecto a la dieta, mientras que en el grupo experimental esta educación verbal se reforzó con folletos de instrucciones con imágenes y una tarjeta de recordatorio para tomar la medicación. Se evaluaron el prurito, el nivel de fósforo en la sangre y los registros de la dieta de cinco días antes y después de la intervención. El grupo experimental mostró un consumo de fósforo en la dieta más bajo que el grupo control $(p<0,001)$. Se observó también una diferencia significativa en el nivel de fósforo en suero entre los grupos experimental y de control $(p=0,002)$. La incidencia de prurito fue menor en el grupo experimental que en el grupo control $(p<0,001)$. Ante estos resultados, este estudio recomienda que las enfermeras incluyan de forma sistemática en la educación de los pacientes el consejo dietético y lo refuercen con imágenes, instrucciones escritas y tarjetas recordatorio para facilitar la toma de la medicación.

Bataille S, Landrier JF, Astier J, Cado S, Sallette $J$, Giaime P, et al. Hemodialysis patients with diabetes eat less than those without: a plea for a permissive diet. Nephrology (Carlton). 2016 Jun 11. doi: $10.1111 /$ nep.12837.

En este estudio se plantea el objetivo de conocer el consumo energético, la ingesta de proteína y de micronutrientes por la dieta, en una población en hemodiálisis. Pretende también determinar qué factores se asocian a un bajo consumo de energía y proteínas y si la ingesta se correlaciona con el estado nutricional.

Se realizó un estudio observacional transversal en una población de pacientes adultos en hemodiálisis, a los que se determinó la ingesta nutricional diaria, la fuerza de empuñadura, la composición corporal medida por bioimpedancia y los parámetros biológicos y de diálisis, incluyendo el Índice de comorbilidad de Charlson.

De los resultados del estudio destaca que la edad mediana de la población estudiada fue de 77.3 [71.1; 84.8] años, que el $57,5 \%$ eran hombres y que el $52,9 \%$ tenían diabetes mellitus.

Respecto a parámetros nutricionales el consumo energético ajustado de peso mediano fue de 18.4 (1078-1569 kcal/día) y la mediana de la ingesta de proteínas por peso ajustado de 0.80 (47.1-66.8 g/ día). En análisis multivariante, ajustado por el peso, el consumo energético fue estadísticamente menor en los pacientes con diabetes $(p=0,01)$ pero no se asoció a otros parámetros. Si el consumo energético no se asoció al peso mediano, la diabetes ya no se asoció a dicho consumo, El género femenino ( $p=$ 0,03 ) y un mayor índice de comorbilidad de Charlson $(p=0,04)$ también se asociaron a un menor consumo de calorías. Respecto la ingesta de proteínas, los resultados fueron similares, y el índice de comorbilidad de Charlson no alcanzó significación estadística.

Los autores concluyen que al tener los pacientes en hemodiálisis un alto riesgo de desnutrición, se les debe prescribir regímenes restrictivos con mucha cautela, especialmente a los diabéticos, pues esta enfermedad es un factor que se asocia significativamente a una baja ingesta dietética. 
Recibido: 25 septiembre 2016

Revisado: 30 septiembre 2016

Modificado: 5 octubre 2016

Aceptado: 6 octubre 2016

\section{Bibliografía}

1. Riobo $P$, Ortiz A. Nutrición en la Insuficiencia renal. Disponible en: http://www.freseniuskabi.es/ pdf/nutri_info/Nutri_Info_05.pdf.

2. Kidney Disease: Improving Global Outcomes (KDIG0) CKD Work Group. KDIGO clinical practice guideline for the evaluation and management of chronic kidney disease. Kidney Int Suppl 2013; 3:1-150. Disponible en: http://www.kdigo. org/clinical_practice_guidelines/pdf/CKD/KDIG0_2012_CKD_GL.pdf.

3. D. de Luis, J. Bustamante. Aspectos nutricionales en la Insuficiencia renal. Nefrología 2008; 28 (3)339-348.

4. Mitch WE, Ikizler TA. Handbook of nutrition and the kidney. 6th ed. Philadelphia, Pa.; London: Lippincott Williams \& Wilkins; 2010.

5. Pérez-Torres A, González E, Bajo MA, Palma Milla $S$, Sánchez Villanueva $R$, et al. Evaluación de un Programa de Intervención Nutricional en enfermedad renal crónica avanzada. Nutr. Hosp. 2013; 28 (6): 2252-2260.

6. Sabatino A, Regolisti G, Karupaiah T, Sahathevan $\mathrm{S}$, Sadu Singh BK, Khor BH et al. Protein-energy wasting and nutritional supplementation in patients with end-stage renal disease on hemodialysis. Clin Nutr. 2016 Jun 18.(16): S0261-5614.

7. Torres MC, Josep $M^{a}$ Gutiérrez, JM, Craver $L$, Baigol MC. Resultado de la intervención Enseñanza: Dieta prescrita en la consulta de enfermedad renal crónica avanzada Enferm Nefrol. 2016;19 (1):12-19. 